\title{
PROCESS AND SYSTEMS Innovate to secure the future: the future of modern medicine
}

\author{
Authors: Sally C Davies ${ }^{A}$ and Claire Oxlade ${ }^{B}$
}

As drug-resistant pathogens emerge and spread globally, antimicrobial (especially antibiotic) treatments are becoming less effective. As infections become more complex and costly to treat in humans and animals, antimicrobial resistance (AMR) is a global challenge of real and increasing scale and complexity. If we do not act for the long term and with sustainability in mind, the annual deaths we see currently, numbering 700,000 globally, will rise each year to 10 million by 2050 . To effectively contain and mitigate AMR, we need more evidence of the drivers and impacts of AMR on human, animal and environmental health, and the links between them. We also need to turn evidence into action and tailored, sustainable approaches for countries and communities, which put clinicians and patients at the centre. Excellent research is underway across the world into innovation (including new treatments, diagnostics and vaccines), infection prevention and behavioural interventions. In this article, we explore how, where and why research should be intensified, with increased collaboration and transparency, to strengthen global health security and secure the future of modern medicine for patients globally.

KEYWORDS: AMR, antimicrobial resistance

DOI: $10.7861 /$ fhj.2021-0087

\section{Introduction}

Antimicrobials, particularly antibiotics, are part of the infrastructure of modern, sustainable healthcare systems. Antibiotics, when appropriately prescribed and equitably accessed, are essential instruments for the healthcare workers that dispense them, and represent a lifeline to patients. Both the restriction and over-consumption of antibiotics, however, contribute to resistance and increased difficulty in treating infections. Driven by natural mutations of microbes, and driving global health insecurity, the post-antibiotic apocalypse is not a vision that is drawn lightly, or without evidence. Antimicrobial

Authors: ${ }^{\mathrm{A} U K}$ special envoy on antimicrobial resistance, Department of Health and Social Care, London, UK; ${ }^{\text {B }}$ private secretary to the UK special envoy on antimicrobial resistance, Department of Health and Social Care, London, UK resistance (AMR) is already a reality for some hospitals and communities, including those individual patients who collectively constitute the mortality figures we have grown accustomed to seeing: 700,000 each year.

The World Health Organization's (WHO) Global action plan on antimicrobial resistance, published in 2015, aims to ensure that, for as long as possible, healthcare workers continue to have the ability to treat and prevent infectious diseases effectively and safely. ${ }^{1}$ Of course, in some countries, this ability is something to be protected; yet, sadly, for some developing countries, resilient, universal health coverage is not yet attained. Local needs, contexts and capabilities impact not only the drivers of AMR, but effective responses to it from healthcare workers in their communities.

In the past year, our global society has experienced the impacts of an initially untreatable infection, COVID-19. Our healthcare systems have faced the repercussions of being reactive and under-prepared. In Spring 2021, 'pandemic preparedness' and 'building back better from COVID-19' are words of the day. These are systemic challenges and opportunities, with solutions based in infrastructure and investment. But this is ultimately about capacity and, particularly, people. Building health systems with agility, quality and public health at the centre depends on keeping the treatments that currently work effective and available, as well as developing new and novel interventions, including diagnostics, vaccines, treatments and alternatives.

Here, we highlight how research and evidence underpin our knowledge of how resistance and transmission occur. Research from social scientists gives insights into people's and populations' understanding of AMR, and how this translates into behaviours that optimise antimicrobial usage and maximises infection prevention. Taken together, evidence can lead to action that directly enables patients to access the right treatments at the right time. We need more of this knowledge, alongside collaboration and transparency. We also need more evidence on the links between AMR and human, animal and environmental health, wellbeing and happiness. We have broad views of the macro-level inequalities and disproportionate impacts of AMR on developing countries, but less so at the micro-level.

The title of our article refers to one of the five priority recommendations put forward by the United Nations (UN) Interagency Coordination Group on AMR, which one author, Prof Dame Sally Davies, co-convened. ${ }^{2}$ Innovation is accelerating to develop new diagnostics, new treatments and even new vaccines to detect, respond and prevent AMR, but we need better 
Box 1. Types of research needed

Discovery science (understanding infectious organisms, their behaviours and impacts on humans, animals, crops and the environment) to find trends of antimicrobial consumption and antimicrobial resistance.

Drivers and transmission of antimicrobial resistance, including understanding of 'spillover', within and between populations, communities or settings, and between humans, animals and the environment.

Innovation (including new treatments, diagnostics, vaccines) and prevention methods (such as infection prevention and control). Clinical research (such as defining optimal dosages and treatment length) and the role of combination treatments. Learning to maintain 'clean' environments (including sewage and manufacturing effluent).

Behavioural understanding to assess the impact of interventions to mitigate antimicrobial resistance, including for effective 'stewardship', communication with patients and the language used to frame and discuss 'antimicrobial resistance' or 'drugresistant infections'.

Implementation and policy research in order to provide local, national and global policy-makers with the evidence and recommendations needed to guide effective actions.

surveillance across the world and deep understanding of the behavioural and social issues underlying AMR too. It is essential that innovation is needs-driven, evidence-based and guided by the principles of affordability, effectiveness, efficiency and equity (Box 1).

COVID-19 will impact policy decisions, funding and research on AMR, though it is as yet unclear whether for better or worse. Ultimately, this is not about competing for agenda space in UN meeting rooms, but about health outcomes and sustainable food systems. To find solutions that prevent AMR and drive appropriate antimicrobial (including antibiotic) use, we need to bring together researchers from across science and medical or veterinary fields, as well as in data science, human behaviour and even anthropology. Significantly, we then need to share research outcomes with clinicians, pharmacists and biomedical engineers as well as with patients and policy makers with feedback to ensure that we research and develop useful new interventions, and ultimately to ensure that they are used appropriately. There is excellent research taking place right across the world on the causes, impacts and mitigations of AMR. There are also many gaps in our knowledge, which we need to fill in order to act effectively; for instance, what impact does AMR in the food chain have on human health? Here, we explore where we are and some of the ongoing research and research gaps.

\section{AMR as a threat multiplier}

AMR is a threat multiplier; at best stagnating recent improvements in oncology care and at worse making chemotherapy prohibitively risky. Physicians know that as many as one in five cancer patients undergoing treatment are hospitalised due to infection, and antibiotics and antifungals are the main lines of defence while their immune systems are weakened by chemotherapy. ${ }^{3}$ Studies suggest that $26.8 \%$ of pathogens that cause infections post-chemotherapy are resistant to standard prophylactic antibiotics. ${ }^{4}$ In short, this

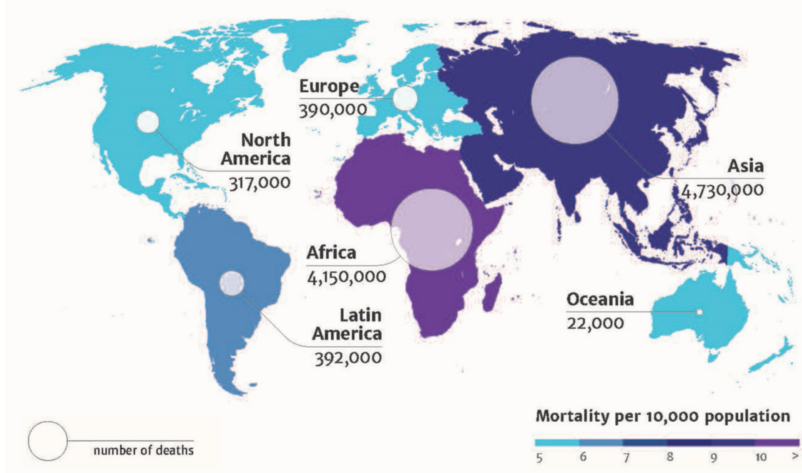

Fig 1. Deaths attributable to antimicrobial resistance every year by 2050. Reproduced with permission from Review on Antimicrobial Resistance. Antimicrobial Resistance: Tackling a Crisis for the Health and Wealth of Nations. Review on Antimicrobial Resistance, 2014.

risks both the safety and efficacy of modern cancer treatment. A recent survey of oncologists in the UK showed that $95 \%$ were worried about the impact of AMR on cancer treatments, with almost half saying that they thought that chemotherapy would soon become unviable. ${ }^{5}$ Almost one-third thought that this would happen within the next 5 years. Modelling suggests that by 2050 , more will die from AMR than from cancer; this is not a competition, but it is a reason for further research into both innovative therapeutics to treat patients with cancer and co-infections, and technology to diagnose infection complications (Fig 1). ${ }^{6,7}$ A general study reviewing the trends and worries of oncologists and oncology patients regarding AMR would be helpful.

AMR underpins a number of global challenges, global systems and, most importantly, the Sustainable Development Goals (SDGs). ${ }^{8}$ The indicators on good health and wellbeing are at risk, as are those on poverty, inequality and hunger. Take HIV and AIDS, where the SDGs progress has been uneven, and we can see a direct link with AMR: in Malawi, research found that $90 \%$ of patients were taking antiretroviral therapy but $80 \%$ of patients with high levels of HIV in their blood had resistance to at least two HIV antiretroviral drugs. These patients were $70 \%$ more likely to die within 2 months of being admitted to hospital than those without drug resistance. ${ }^{9}$ The London School of Hygiene and Tropical Medicine study demonstrates how AMR is undermining antiretroviral treatment, how important it is to quickly diagnose drug resistance in HIV patients and move to alternative treatments. Stigma and marginalisation stops entire communities' access to health systems, education or services, speaking to why HIV (and AMR) is also about access to treatments.

There is emerging evidence from around the world that COVID-19 is exacerbating unnecessary prescribing of antibiotics. A study primarily focused on China, the USA, Italy and South Korea during the first major wave of the pandemic, up to June 2020 , indicated that around $75 \%$ of COVID-19 patients received antibiotics, despite only $3.5 \%$ and $14.3 \%$ of patients being hospitalised for bacterial co-infection and secondary infection, respectively. ${ }^{10}$ This trend of over prescribing was also reflected in community settings, where primary care prescription messaging indicated that the number of prescriptions commonly used for respiratory infections was $44 \%$ higher in the fourth week of March 
2020 than the same week in 2019.11 This increased use of broadspectrum antimicrobials for COVID-19 patients, compounded by the increased reliance on telehealth services, suggests that inappropriate prescribing could have a significant unintended longer-term impact on AMR.

Notably though, this 'surge' in community prescribing preceded a fall in April and May 2020, with prescriptions 34\% lower than the same week in 2019. ${ }^{11}$ It is possible that non-pharmaceutical measures to tackle COVID-19, such as wearing masks and social distancing mitigated infections and, therefore, the need for antibiotics. If these behaviours become entrenched, we could even see benefits for AMR. An analysis on whether prescribing levels were influenced more by patients choosing to provide self-care as directed by NHS websites, by patient 'fear' of adding to pressures to the NHS or more from beneficial decisions made by healthcare workers. To ensure that patients receive the best treatments (both for COVID-19 and for all infections during pandemics), we need to understand both what is happening and the underlying reasons. To help assess the impact of COVID-19 on AMR, the University of Oxford is now started a cohort study that will use clinical patient data from hospitals across 11 countries (including Italy, the UK, India, Korea and Brazil) focusing on changes in antibiotic usage and stewardship, and also on resistance. ${ }^{12}$

\section{The scale of AMR}

Currently, the global scale of AMR and antimicrobial use (AMU) is not fully understood. This means that local guidelines and interventions can be tricky to negotiate. The WHO's Global Antimicrobial Resistance Surveillance System (GLASS) seeks to harmonise surveillance from over 65,000 surveillance sites from 66 countries for eight priority human pathogens. ${ }^{13}$ Data submission from Africa and South-East Asia has increased but there will need to be a greater effort to ensure that the data is representative of all countries and communities. Using clinical outcome data would also mark a useful step change in the global surveillance picture.

The UK's Fleming Fund is a $£ 265$ million UK aid programme supporting up to 24 countries across Africa and Asia to strengthen surveillance systems to understand and tackle AMR. The Fleming Fund is investing in countries' clinical microbiology capacity to carry out their own surveillance, including submitting data to GLASS. Similarly, the US Centers for Disease Control and Prevention (CDC) aims to expand its Antibiotic Resistance Lab Network internationally so that networks can detect, contain, test and respond to AMR. ${ }^{14}$ The CDC is already working in Kenya to build a laboratory-based surveillance network, which will eventually also contribute to global surveillance systems run by WHO. The US investment in expanding their international surveillance could be a gamechanger. We all recognise that the more we know, the better we can tackle AMR globally.

Many low- and middle-income countries (LMICs) lack surveillance capacity, so proxies and modelling are building our knowledge. A world-first study funded by the UK's Fleming Fund, the Wellcome Trust and the Bill and Melinda Gates Foundation used geostatistical modelling from household surveys estimate global antibiotic consumption across 204 countries. ${ }^{15}$ Between 2000 and 2018 , antibiotic consumption increased by an average of $46 \%$, with the Philippines consuming 9.18 times fewer antibiotics than Greece in 2018. This demonstrates the global imbalance between high consumption and lack of access to antibiotics; for example, WHO's GLASS indicated that only $27.3 \%$ of primary treatments complied with national treatment guidelines in the Philippines for gonorrhoea. $^{16}$

To reconcile the access versus excess problem, we need to fix the underlying problems: the high up-front costs and the low prices that health systems pay that lead pharmaceutical companies to disinvest from antibiotic research and development. This leaves too few novel antibiotic and other antimicrobial treatments in the pipeline and no new class of antibiotics available to the market this side of the millennium. We need innovation, particularly to ensure that late-stage development for novel antibiotics is feasible. An analysis of European public funding indicated that $86 \%$ of national-level funding for antibiotics was directed at basic research, leaving a glaring gap for the small and medium-sized enterprises who develop this research into usable, and potentially accessible, products. ${ }^{17}$

\section{Positive steps and future opportunities}

Last year, the UK became the first country in the world to launch a new system that will trial paying pharmaceutical companies for novel antimicrobials based on their value to our NHS and wider society. ${ }^{18}$ This is a world-first experiment based on policy research so the NHS in England can trial paying for antibiotics by subscription, rather than paying for the quantity used. The objective of this so-called 'Netflix model' is to empower clinicians to follow appropriate stewardship to maximise the benefit to patients, while encouraging companies to invest in the effective antibiotics that patients need. The selection process was designed to favour products that address an infection area of key importance internationally and, specifically, address a key unmet need in the UK (such as products which are effective against resistant Gram-negative pathogens in high-risk settings). Points were also awarded during procurement for activity against the WHO 'priority pathogens' list, as well as for the supplier's demonstrated commitment to antimicrobial and environmental stewardship, and robust plans for surveillance. Following a rigorous process with expert clinical input, two therapies, cefiderocol (Fetcroja; manufactured by Shionogi) and ceftazidime with avibactam (Zavicefta; manufactured by Pfizer) are now moving through an innovative health technology evaluation process. ${ }^{19}$ The output of these evaluations will inform the subscription payment price each company will receive for its product and could bring two new antibiotics to patients by 2022.

Policy initiatives from governments do not work in silos but work in the ecosystem that brings together healthcare systems, researchers and pharmaceutical companies. The new AMR Action Fund, launched last summer, is an industry-led initiative, supported by the International Federation of Pharmaceutical Manufacturers \& Associations, the Wellcome Trust and the European Investment Bank, and over 20 leading pharmaceutical companies, to bring between two to four new antibiotics to market. ${ }^{20}$ This represents a bridge between research and patient access. When combined with pull incentives from governments (like the UK's Netflix model), this should revitalise the pipeline of treatments available for healthcare workers to use.

Innovation depends on action and collaboration across sectors and healthcare settings. Global non-profit partnerships (such as (ARB-X) are funding early stage research to develop new treatments, particularly for use in LMICs. Targeting neglected and under-invested areas of research and development (R\&D), CARB-X supports projects through the tricky early development phases, 
including recently awarding an initial $£ 2$ million to the Jenner Institute at University of Oxford to develop a novel vaccine to prevent multi-drug resistant Neisseria gonorrhoeae. ${ }^{21}$ Gonorrhoea is one of WHO's priority pathogens which disproportionately impacts women in developing countries, this novel vaccine aims to be affordable and ensure that we can reserve the single remaining class of antibiotics that are effective against newly resistant strains of gonorrhoea. Vaccines are sustainable policy interventions in their own right to mitigate AMR, which are sustainable and, thanks to multilateral organisations like Gavi, The Vaccine Alliance, broadly accessible. Biological resistance to vaccines is also rare, though we need to ensure that we monitor emerging global trends.

Innovation and access go hand-in-hand. The Wellcome Trust's ABACUS project was conducted in a semi-rural district of southern Mozambique. It explored participants' knowledge of antibiotics and worked out how participants recognised antibiotics from their visual appearance (based on colours and shapes), in this way helping guide future use. ${ }^{22}$

CARB-X has developed a new stewardship and access plan so that developers will be guided to ensure that their products are accessible and available. ${ }^{23}$ This guide, therefore, is designed to encourage developers to think practically and early about how their products will be used in real health settings. This can include guidance on pricing, distribution and even packaging ensuring that healthcare workers and patients of all languages and literacy levels are well supported to understand product labels. As product developers and as product prescribers, we clearly all need to take the everyday experience of patients into account and develop the best channel of education for them.

Innovation is especially needed for paediatric practice. While we know that annually approximately 214,000 neonatal deaths are caused by drug-resistant infections, to date there has been little research on the use and duration of antibiotic treatment in them. ${ }^{24}$ The Access to Medicine Foundation point to a paucity of R\&D projects for children under 12 years old, with only 53 out of 1,073 projects analysed specifically for children under 5 years old, despite being the ages of the majority of paediatric deaths. ${ }^{25}$ So now an observational study to collect clinical data from neonatal units from 11 countries across four continents is seeking to generate data that will inform future interventions on new-borns with sepsis. This study will influence both the Global Antibiotic Research and Development Partnership programme to develop new antibiotic treatments specifically for newborn babies, and global efforts on diagnostics and detection of hospitalacquired infections. ${ }^{26}$ Yet, the access versus excess dichotomy is exacerbated in paediatric care, including in the UK. Research led by King's College London indicated that age-band dosing guidelines have remained unchanged since 1963 and there is wide variation in prescribing practice, with the majority of children receiving sub-optimal doses. National prescribing guidelines and practice have been changed as a result, with now $94 \%$ of children receiving the optimal dose for their age, weight and condition. As of 2019, these guidelines have been adapted to local prescribing guidelines in over 40 countries. ${ }^{27}$

To effect sustainable action, healthcare workers need to bring the public along in the journey from infection prevention to health promotion. So, children are central to AMR, not just as patients but as social citizens and youth advocates. Behavioural interventions aimed at increasing awareness and education of AMR among children have been demonstrably successful. The Mould that
Changed the World is a musical aimed to engage primary schoolaged children on the importance of using antibiotics appropriately. A quantitative study on the knowledge gain by children participants indicated that key messages were remembered in both the short and long term. ${ }^{28}$

\section{Bridging evidence and action on AMR}

AMR does not stand still: it evolves, and research needs to evolve with it. We need to equip clinicians to be able to respond to the present threats in front of them. Thanks to UK investments in genome sequencing capacity in South Africa, scientists were able to identify a new SARS-CoV-2 variant, which is influencing the global response. ${ }^{29}$ Through sharing data, we can make our investments in surveillance and research go further and build on previous efforts. The Pew Foundation's Shared Platform for Antibiotic Research and Knowledge (SPARK) is an excellent example of a tool to pool insights and data and enable realtime collaboration between academia, industry, clinicians and governments. ${ }^{30}$ The database focuses on how Gram-negative bacteria spread, so that innovators can concentrate their efforts on the present mutations. Open research should be a means, not the end, to enabling access to medicines. The 2020 AMR Benchmark published by Access to Medicine Foundation (ATMF) ranked companies for their initiatives to share intellectual capital. ${ }^{31}$ ATMF noted that Entasis and GlaxoSmithKline were leaders in sharing intellectual property with third-party researchers, which ultimately stimulates R\&D and surmounts barriers to access. More, open research will make the case for more countries to prioritise AMR with policies and funding.

According to a global survey conducted by the UN, universal access to healthcare was ranked as the top short-term priority, with greater environmental protection highlighted as the biggest long-term priority. ${ }^{32}$ There is now emerging evidence of the links between AMR and climate change, with waterways acting as both a vector of transmission for $\mathrm{AMR}$, and as the victim of pollution from antibiotic manufacturing and discharge. In Australia, scientists have studied the presence of a type of resistant bacteria, Enterobacteriales, in green sea turtles on the Great Barrier Reef. They found that $37.7 \%$ of the bacterial samples they studied were identified as multi-drug resistant. ${ }^{33}$

We need more research, evidence and monitoring of the impact of AMR on climate change, and vice versa, including evidence on what interventions would be most helpful. The Dasgupta review, the first comprehensive economic framework for biodiversity, calls for transformative change in how we think, act and measure economic success so that it protects the natural world. ${ }^{34}$ That is why the UK's antibiotic purchasing pilot includes environmental standards in its evaluation of the value of new antibiotic contracts. Research analysis on the water quality around Caithness General Hospital in the Scottish Highlands identified paracetamol and carbamazepine in wastewater samples, reflecting the medicines used frequently in the hospital. ${ }^{35}$ We know antibiotics leak through general sewage into rivers and water tables too. Clinicians in that NHS region now prescribe from a designated list of environmentally friendly medicines, showing the importance of environment health and human health but more work is needed on AMR.

Ultimately, we need to turn the numbers into meaningful, sustainable and local action. With bottom-up, community-led 
approaches that put patients and prescribers first, we can build resilient local healthcare systems; for example, the Commonwealth Partnerships for Antimicrobial Stewardship Brighton-Lusaka partnership produced a wonderful educational video teaching local pharmacy teams how to make hand sanitiser, sharing and upskilling is vital as we learn more about infections through evidence. ${ }^{36}$ By translating research into daily practice, we can use evidence for action, whether in communities, hospitals or by governments.

Only through research can we find out what works (and what does not) to shape our future health systems. In Australia, letters were sent to general practice prescribers with high prescribing rates, that compared them with other local prescribers and helped to benchmark more 'appropriate' prescribing levels. Within 6 months, there was a $9 \%-12 \%$ reduction compared with the control group. ${ }^{37}$ But this is context specific, and even patient specific, and is unlikely to be the answer for every setting. New and novel approaches depend on innovative and imaginative researchers.

\section{Conclusion}

2021 is already shaping up to be an important year for global health, animal health and environmental policy. This year, the UK will host COP26 and hosts the G7 presidency: both opportunities to work with international partners to build back better from COVID-19. AMR will be a shared priority for governments, to strengthen global health security and prevent future crises. We will be pushing for more research and surveillance, and the better stewardship of existing antibiotics. We must reinvigorate the development of new treatments, diagnostics and vaccines too, while making sure the antibiotic supply chain is safe, secure and transparent, and has shared standards that we can all rely upon. Under the UK's leadership, the $\mathrm{G} 7$ will strengthen $\mathrm{R} \& \mathrm{D}$ innovation and patient access for the whole world.

Research happens in many ways and is conducted by many different people. It is also used by many people in different ways, whether in laboratories to develop new medicines, or in hospitals and community care settings to ensure that the right treatments reach the right patients at the right time. By collaborating across sectors and countries, we can innovate to secure the future of modern medicine, and not move into a post-antibiotic apocalypse.

\section{References}

1 World Health Organization. Global action plan on antimicrobial resistance. WHO, 2015. www.who.int/antimicrobial-resistance/ global-action-plan

2 Interagency Coordination Group on AMR. No time to wait: Securing the future from drug-resistant infections. WHO, 2019. www.who.int/antimicrobial-resistance/interagency-coordinationgroup/IACG_final_report_EN.pdf?ua=1

3 AMR Insights. Antimicrobial resistance and its impact on cancer care. AMR Insights, 2020. www.amr-insights.eu/antimicrobialresistance-and-its-impact-on-cancer-care

4 Bisson JL, Argyle SA. Antibiotic prophylaxis in veterinary cancer chemotherapy: A review and recommendations. Veterinary and Comparative Oncology 2018;16:301-310.

5 Longitude Prize. Effectiveness of cancer treatments threatened by rising antibiotic resistance. Longitude Prize, 2020. https://longitudeprize.org/resources/effectiveness-of-cancer-treatments-threatenedby-rising-antibiotic-resistance
6 Review on Antimicrobial Resistance. Tackling drug-resistant infections globally: final report and recommendations. Review on Antimicrobial Resistance, 2016. https://amr-review.org/sites/ default/files/160525_Final\% 20paper_with\% 20cover.pdf

7 Review on Antimicrobial Resistance. Antimicrobial Resistance: Tackling a Crisis for the Health and Wealth of Nations. Review on Antimicrobial Resistance, 2014. https://amr-review.org/sites/ default/files/AMR \% 20Review \% 20Paper \% 20- \% 20Tackling \% 20 a $\% 20$ crisis $\% 20$ for $\% 20$ the $\% 20$ health $\% 20$ and $\% 20$ wealth $\% 20$ of $\%$ 20nations_1.pdf

8 ReAct. Tracking antimicrobial resistance in the Sustainable Development Goals. ReAct, 2019. www.reactgroup.org/news-andviews/news-and-opinions/year-2019/tracking-antimicrobial-resistance-in-the-sustainable-development-goals

9 Gupta-Wright A, Fielding K, van Oosterhout J] et al. Virological failure, HIV-1 drug resistance, and early mortality in adults admitted to hospital in Malawi: an observational cohort study. Lancet HIV 2020;7:e620-8.

10 Langford B], So M, Raybardhan S et al. Bacterial co-infection and secondary infection in patients with COVID-19: a living rapid review and meta-analysis. CMI 2020;26:P1622-9. www. clinicalmicrobiologyandinfection.com/article/S1198743X(20)30423-7/fulltext

11 Malcolm W, Seaton RA, Haddock G et al. Impact of the COVID-19 pandemic on community antibiotic prescribing in Scotland. JAC Antimicrob Resist 2020;2:dlaa105.

12 Buckland Merrett G. Could Covid-19 be fuelling drug resistance? Wellcome Trust, 2020. https://wellcome.org/news/couldcovid-19-be-fuelling-drug-resistance

13 World Health Organization. Global Antimicrobial Resistance Surveillance System (GLASS). WHO. www.who.int/glass/en

14 Federal Task Force on Combating Antibiotic-Resistant Bacteria. National action plan for combating antibiotic-resistant bacteria, 2020-2025. Office of the Assistant Secretary for Planning and Evaluation, 2020. https://aspe.hhs.gov/system/files/pdf/264126/ CARB-National-Action-Plan-2020-2025.pdf

15 Browne AJ, Chipeta MG, Haines-Woodhouse G et al. Global antibiotic consumption in humans, 2000 to 2018: a spatial modelling study. [Non-reviewed preprint, 2020]. https://papers.ssrn.com/sol3/ papers.cfm?abstract_id $=3746925$

16 World Health Organization. Global Antimicrobial Resistance and Use Surveillance System (GLASS) Report. WHO, 2020. https://apps. who.int/iris/bitstream/handle/10665/332081/9789240005587eng.pdf

17 Simpkin VL, Renwick MJ, Kelly R, Mossialos E. Incentivising innovation in antibiotic drug discovery and development: progress, challenges and next steps. The Journal of Antibiotics 2017;70:108796. www.nature.com/articles/ja2017124

18 Department of Health and Social Care. World-first scheme underway to tackle AMR and protect UK patients. DHSC, 2020. www.gov.uk/government/news/world-first-scheme-underway-totackle-amr-and-protect-uk-patients

19 Perkins M, Glover D. How the 'NHS model' to tackle antimicrobial resistance (AMR) can set a global standard. NHS, 2020. www. england.nhs.uk/blog/how-the-nhs-model-to-tackle-antimicrobialresistance-amr-can-set-a-global-standard

20 AMR Action Fund. Antimicrobial Resistance Research \& Development. AMR Action Fund. https://amractionfund.com

21 CARB-X. CARB-X is funding University of Oxford's Jenner Institute to develop a new vaccine to prevent gonorrhea. CARB-X. https://carb-x.org/carb-x-news/carb-x-is-fundinguniversity-of-oxfords-jenner-institute-to-develop-a-newvaccine-to-prevent-gonorrhea/\#: :text=CARB-X\% 20is \% 20 funding $\%$ 20University $\%$ 20 of $\%$ 200xford $\%$ E2 \% 80 \% 99s \% 20 Jenner $\%$ 20Institute,disease $\%$ 20and $\%$ 20strains \% 20 of \% 20 \% E2 \% 80\% 98super \% 20gonorrhea \% E2 \% 80\% 99\% 20antibioticresistant \% 20bacteria 
22 Cambaco O, Alonso Menendez Y, Kinsman ] et al. Community knowledge and practices regarding antibiotic use in rural Mozambique: where is the starting point for prevention of antibiotic resistance? BMC Public Health 2020:20:1183.

23 CARB-X. Stewardship and Access Plan Development Guide provides a new benchmark for product developers around the world. CARBX. https://carb-X.org/about/stewardship-and-access/\#: :text=CARBX-funded \% 20product \% 20developers \% 20are \% 20contractually $\%$ 20obligated $\%$ 20to \% 20develop,appropriate $\% 20$ access $\% 20$ in $\%$ 20low- $\%$ 20and $\% 20$ middle-income $\% 20$ countries $\% 20 \% 28$ LMICs $\% 29$

24 Laxminarayan R, Matsoso P, Pant S et al. Access to effective antimicrobials: a worldwide challenge. Lancet 2016;387:168-75.

25 Access to Medicine Foundation. Handful of new children's meds on the horizon. But will they be accessible to children in LMICs? Access to Medicine Foundation, 2021. https://accesstomedicinefoundation.org/news/handful-of-new-childrens-meds-on-the-horizon-butwill-they-be-accessible-to-children-in-Imics

26 Global Antibiotic Research \& Development Partnership. Researchers gather in New Delhi to kick off observational study for newborns with sepsis. GARDP, 2018. www.gardp.org/news-resources/ researchers-gather-in-new-delhi-to-kick-off-observational-study-fornewborns-with-sepsis

27 Saxena S, Ismael Z, Murray ML et al. Oral penicillin prescribing for children in the UK: a comparison with BNF for Children age-band recommendations. Br J Gen Pract 2014;64:e217-22.

28 Hall J, Jones L, Robertson G et al. "The Mould that Changed the World": a quantitative and qualitative evaluation of knowledge and behavioural change in children in the UK following participation in a musical about antimicrobial resistance. Lancet 2019;394:S47.

29 The Fleming Fund. Genomics, the Fleming Fund and COVID-19. The Fleming Fund, 2020. www.flemingfund.org/publications/ genomics-the-fleming-fund-and-covid-19

30 PEW. The Shared Platform for Antibiotic Research and Knowledge (SPARK). PEW, 2021. www.pewtrusts.org/en/research-and-analysis/ articles/2018/09/21/the-shared-platform-for-antibiotic-researchand-knowledge
31 Access to Medicine Foundation. More pharma companies join the leaders in 2020, yet progress on AMR is slow. Access to Medicine Foundation, 2020. https://accesstomedicinefoundation.org/amrbenchmark

32 United Nations. The future we want, the United Nations we need. UN, 2020. www.un.org/sites/un2.un.org/files/un75report_ september_final_english.pdf

33 Frade PR, Glasl B, Matthews SA et al. Spatial patterns of microbial communities across surface waters of the Great Barrier Reef. Communications Biology 2020;3:442.

34 Dasgupta P. The economics of biodiversity: the Dasgupta review. London: HM Treasury, 2021. https://assets.publishing.service. gov.uk/government/uploads/system/uploads/attachment_data/ file/962785/The_Economics_of_Biodiversity_The_Dasgupta_ Review_Full_Report.pdf

35 Niemi L, Taggart M, Boyd K et al. Assessing hospital impact on pharmaceutical levels in a rural 'source-to-sink' water system. Science of The Total Environment 2020;737:139618.

36 Commonwealth Pharmacists Association. Press Release: How to Manufacture Alcohol Hand Rub - Training video launched to support COVID-19 response. Commonwealth Pharmacists Association, 2020. https://commonwealthpharmacy.org/press-release-how-tomanufacture-alcohol-hand-rub-training-video-launched-to-supportcovid-19-response

37 Hendrie D. 'Nudge' letters prompt sustained drop in antibiotic prescribing. Royal Australian College of General Practitioners, 2020. www1.racgp.org.au/newsgp/professional/nudge-letters-promptsustained-drop-in-antibiotic

Address for correspondence: Prof Dame Sally Davies, Department of Health and Social Care, 39 Victoria Street, London SW1H OEU, UK.

Email: amrenvoy@dhsc.gov.uk

Twitter: @UKAMREnvoy 